\title{
Every non-Euclidean oriented matroid admits a biquadratic final polynomial
}

\section{Journal Article}

Author(s):

Fukuda, Komei; Moriyama, Sonoko; Nakayama, Hiroki; Richter-Gebert, Jürgen

Publication date:

2009

Permanent link:

https://doi.org/10.3929/ethz-b-000018141

Rights / license:

In Copyright - Non-Commercial Use Permitted

Originally published in:

Combinatorica 29(6), https://doi.org/10.1007/s00493-009-2255-2 


\title{
EVERY NON-EUCLIDEAN ORIENTED MATROID ADMITS A BIQUADRATIC FINAL POLYNOMIAL
}

\author{
KOMEI FUKUDA*, SONOKO MORIYAMA, HIROKI NAKAYAMA, \\ JÜRGEN RICHTER-GEBERT
}

\author{
Received October 31, 2005 \\ Revised June 13, 2007
}

Richter-Gebert proved that every non-Euclidean uniform oriented matroid admits a biquadratic final polynomial. We extend this result to the non-uniform case.

\section{Introduction}

In this paper, we identify a chirotope $\chi$ with an oriented matroid $\mathcal{M}=(E, \chi)$, which we abbreviate by OM. A standard reference for the theory of oriented matroids is [2]. The set $E=\{1, \ldots, n\}$ is called ground set and $\chi: E^{r} \rightarrow$ $\{+1,-1,0\}$ satisfies chirotope axioms, where $r$ is a rank of an OM and $n$ is a number of elements of the ground set.

Let $X=\left(x_{1}, \ldots, x_{n}\right) \in \mathbb{R}^{r \times n}$ be a configuration of $n$ points in $\mathbb{R}^{r}$. Let $\left[i_{1} \cdots i_{r}\right]$ denote the determinant $\operatorname{det}\left(x_{i_{1}} \cdots x_{i_{r}}\right)$. By setting $\chi_{X}\left(i_{1}, \ldots, i_{r}\right)=$ $\operatorname{sgn}\left[i_{1} \cdots i_{r}\right]$, the function $\chi_{X}$ satisfies the chirotope axioms. A chirotope arising this way is called representable or realizable. It is well known that not all chirotopes are realizable.

In the sequel, we regard a bracket $\left[i_{1} \cdots i_{r}\right]$ as a bracket variable. For any given ordered sequences of indices $\tau=\left(\tau_{1} \cdots \tau_{r-2}\right)$ and $\lambda=\left(\lambda_{1} \cdots \lambda_{4}\right)$, we call a bracket polynomial

$$
\left[\tau \lambda_{1} \lambda_{2}\right]\left[\tau \lambda_{3} \lambda_{4}\right]-\left[\tau \lambda_{1} \lambda_{3}\right]\left[\tau \lambda_{2} \lambda_{4}\right]+\left[\tau \lambda_{1} \lambda_{4}\right]\left[\tau \lambda_{2} \lambda_{3}\right]
$$

Mathematics Subject Classification (2000): 52C35, 52C40

* Research supported by the Swiss National Science Foundation Project 200021-105202, "Polytopes, Matroids and Polynomial Systems". 
a 3-term Grassmann-Plücker polynomial. If the chirotope is realizable, the value of (1) is always 0 . Now we introduce biqudratic inequalities (equations) and define biquadratic final polynomials.

Definition 1.1. Let $\chi$ be an OM of rank $r$, let $\tau \in E^{r-2}, \lambda \in E^{4}$ be index sequences, and let $A=\left(\tau \lambda_{1} \lambda_{2}\right), B=\left(\tau \lambda_{3} \lambda_{4}\right), C=\left(\tau \lambda_{1} \lambda_{3}\right), D=\left(\tau \lambda_{2} \lambda_{4}\right)$, $E=\left(\tau \lambda_{1} \lambda_{4}\right)$ and $F=\left(\tau \lambda_{2} \lambda_{3}\right)$. Then

1. A pair $(\tau, \lambda)$ is called $\chi$-normalized if $\chi(A) \cdot \chi(B) \geq 0, \chi(C) \cdot \chi(D) \geq 0$ and $\chi(E) \cdot \chi(F) \geq 0$.

2. For a $\chi$-normalized pair $(\tau, \lambda)$, we call

$[A][B]<[C][D]$ and $[E][F]<[C][D] \quad$ biquadratic inequalities, $[A][B]=[C][D] \quad$ or $\quad[E][F]=[C][D]$ a biquadratic equation.

We remark that for any pair $(\tau, \lambda)$, by permutating $\left(\lambda_{1}, \lambda_{2}, \lambda_{3}, \lambda_{4}\right)$ appropriately, $(\tau, \lambda)$ becomes $\chi$-normalized. We denote the set of biquadratic inequalities and biquadratic equations by $\mathcal{A}_{\chi}$ and $\mathcal{B}_{\chi}$, respectively. If $\chi$ is uniform, $\mathcal{B}_{\chi}=\emptyset$.

Definition 1.2. An OM $\chi$ is said to admit a biquadratic final polynomial if there are a non-empty subset of $\mathcal{A} \chi:\left\{\left[A_{i}\right]\left[B_{i}\right]<\left[C_{i}\right]\left[D_{i}\right] \mid 1 \leq i \leq k\right\}$ and a (possibly empty) subset of $\mathcal{B}_{\chi}:\left\{\left[A_{j}\right]\left[B_{j}\right]=\left[C_{j}\right]\left[D_{j}\right] \mid 1 \leq j \leq l\right\}$ such that the following equality holds

$$
\prod_{i=1}^{k}\left[A_{i}\right]\left[B_{i}\right] \cdot \prod_{j=1}^{l}\left[A_{j}\right]\left[B_{j}\right]=\prod_{i=1}^{k}\left[C_{i}\right]\left[D_{i}\right] \cdot \prod_{j=1}^{l}\left[C_{j}\right]\left[D_{j}\right] .
$$

The following is a direct consequence of the definition above.

Lemma 1.3. If $\chi$ admits a biquadratic final polynomial, $\chi$ is non-realizable.

Richter-Gebert [6] proved that every non-Euclidean uniform oriented matroid admits a biquadratic final polynomial. Our main theorem extends this result to the non-uniform case.

Theorem 1.4. Every non-Euclidean oriented matroid admits a biquadratic final polynomial.

\section{Oriented Matroid Programming}

Oriented matroid programming is formulated as a combinatorial abstraction of linear programming [1]. The simplex method in linear programming has a natural extension in the setting of oriented matroids. Edmonds and 
Fukuda [3] showed that there exist OMs allowing the simplex method to generate a cycle of non-degenerate pivots, which cannot ${ }^{1}$ occur in linear programming. Consequently, one can show the non-realizability of an OM by exhibiting a non-degenerate cycle of simplex pivots if exists.

Let $\chi$ be an OM of rank $r$ on an $(n+2)$ element set $E=\{1, \ldots, n, f, g\}$. Here, the last two elements $f$ and $g$ of $E$ are distinguished. The triple $(\chi, f, g)$ is called an oriented matroid program (abbreviated by OMP). The element $g$ represents a hyperplane at infinity and $f$ represents an objective function.

Definition 2.1. Let $(\chi, f, g)$ be an OMP and $\mathcal{A}\left(\mathcal{A}^{\infty}\right.$, respectively) be the affine (infinite) space with respect to $g$, i.e., the set of covectors with positive (zero) $g$-component.

1. A set $B=\left(\lambda_{1}, \ldots, \lambda_{r-1}\right) \in E-\{f, g\}$, such that $B \cup\{g\}$ is independent, is called an affine basis. The unique vertex (i.e., a covector with minimal support, or equivalently a cocircuit) $X$ with $X_{B}=0$ and $X_{g}=+$ is denoted by $v(B)$.

2. $B_{1} \rightarrow B_{2}$ is called a pivot operation if $B_{1}, B_{2}$ are affine bases and $L=$ $B_{2}-\{b\}=B_{1}-\{a\}$ where $a, b \in E-\{f, g\}$ and $a \neq b . L$ is called the edge of $B_{1} \rightarrow B_{2}$.

3. The direction of a pivot $L \cup\{a\}=B_{1} \rightarrow B_{2}=L \cup\{b\}$ where $L \cup\{a, b\}$ is assumed to be independent, is the unique vertex $d=d\left(B_{1} \rightarrow B_{2}\right) \in \mathcal{A}^{\infty}$ with $d_{L}=0$ and $d_{a}=v\left(B_{2}\right)_{a}$.

4. A pivot operation $L \cup\{a\}=B_{1} \rightarrow B_{2}=L \cup\{b\}$ where $a \neq b$ is called degenerate if $v\left(B_{1}\right)=v\left(B_{2}\right)$,

horizontal if $L \cup\{f, g\}$ is dependent, strictly increasing if $d\left(B_{1} \rightarrow B_{2}\right)_{f}>0$ and $B_{1} \rightarrow B_{2}$ is not degenerate.

We remark that neither degenerate nor horizontal pivot operation occurs when an OM $\chi$ is uniform.

Definition 2.2. A sequence of pivot operations $B_{1} \rightarrow B_{2} \rightarrow \cdots \rightarrow B_{k}$ is called a non-degenerate cycle on $\chi$ if $B_{1}=B_{k}$ and all pivot operations are either degenerate, horizontal or strictly increasing and at least one pivot is strictly increasing.

Since no non-degenerate cycling occurs in linear programming, the following proposition holds.

Proposition 2.3. If an $O M P(\chi, f, g)$ admits a non-degenerate cycle, then the oriented matroid $\chi$ is non-realizable.

\footnotetext{
${ }^{1}$ Note that in linear programming, the simplex method can generate a cycle of degenerate pivots, known as cycling.
} 
The following characterization of Euclidean OMs is fundamental.

Proposition 2.4 ([3]). An $O M P(\chi, f, g)$ on $E$ admits a non-degenerate cycle for some choice of two distinguished elements $f$ and $g$ from $E$ if and only if the oriented matroid $\chi$ is non-Euclidean.

\section{From Cycling to Biquadratic Final Polynomial}

In the case of uniform OMs, Richter-Gebert [6] gave a method to obtain a biquadratic final polynomial from a non-degenerate cycle. Now we extend this method to the non-uniform case. In the following proof, we translate each pivot operation to one Grassmann-Plücker polynomial.

Lemma 3.1. Let $(\chi, f, g)$ be an $O M P$ and $L=\left\{\lambda_{1}, \ldots, \lambda_{r-2}\right\} \subset E-\{f, g\}$, $a, b \in E-\{f, g\}$ such that $L \cup\{a\}=B_{1} \rightarrow B_{2}=L \cup\{b\}$ is a pivot operation along edge $L$. Then

- if $B_{1} \rightarrow B_{2}$ is strictly increasing, $\chi\left(\lambda_{1}, \ldots, \lambda_{r-2}, g, f\right) \cdot \chi\left(\lambda_{1}, \ldots, \lambda_{r-2}, a, b\right)$. $\chi\left(\lambda_{1}, \ldots, \lambda_{r-2}, g, a\right) \cdot \chi\left(\lambda_{1}, \ldots, \lambda_{r-2}, g, b\right)=+1$,

- if $B_{1} \rightarrow B_{2}$ is either degenerate or horizontal, $\chi\left(\lambda_{1}, \ldots, \lambda_{r-2}, g, f\right)$. $\chi\left(\lambda_{1}, \ldots, \lambda_{r-2}, a, b\right) \cdot \chi\left(\lambda_{1}, \ldots, \lambda_{r-2}, g, a\right) \cdot \chi\left(\lambda_{1}, \ldots, \lambda_{r-2}, g, b\right)=0$.

Proof. For the first case, see [6]. If the pivot operation is degenerate, which means two affine vertices $v\left(B_{1}\right)$ and $v\left(B_{2}\right)$ are at the same point, $\chi\left(\lambda_{1}, \ldots, \lambda_{r-2}, a, b\right)=0$. Similary, if the pivot operation is horizontal, that is $L \cup\{f, g\}$ is dependent, $\chi\left(\lambda_{1}, \ldots, \lambda_{r-2}, f, g\right)=0$ is satisfied. For both two cases, the values become 0 .

We are now ready to prove the main theorem.

Proof of Theorem 1.4. Let $\chi$ be a non-Euclidean OM on E. By Proposition 2.4, there exist $f$ and $g$ in $E$ such that the $\operatorname{OMP}(\chi, f, g)$ admits a non-degenerate cycle, say, $B_{1} \rightarrow B_{2} \rightarrow \cdots \rightarrow B_{k}$ where $B_{1}=B_{k}$. We shall construct a suitable biquadratic final polynomial. We define $L^{i}, a^{i}, b^{i}$ by the relations:

$$
L^{i} \cup\left\{a^{i}\right\}=B_{i} \rightarrow B_{i+1}=L^{i} \cup\left\{b^{i}\right\} \quad \text { for all } 1 \leq i \leq k .
$$

In (2), we set $B_{k+1}=B_{2} . L^{i}=\left\{\lambda_{1}^{i}, \ldots, \lambda_{r-2}^{i}\right\}$ is the edge of the pivot operation $B_{i} \rightarrow B_{i+1}$. We denote $\lambda^{i}=\left(\lambda_{1}^{i}, \ldots, \lambda_{r-2}^{i}\right)$. Consider the following sequence of Grassmann-Plücker polynomials:

$$
G P^{i}=\left[\lambda^{i}, g, f\right]\left[\lambda^{i}, a^{i}, b^{i}\right]-\left[\lambda^{i}, g, a^{i}\right]\left[\lambda^{i}, f, b^{i}\right]+\left[\lambda^{i}, g, b^{i}\right]\left[\lambda^{i}, f, a^{i}\right] .
$$


Note that $G P^{1}=G P^{k}$. As in Definition 1.1, we set $A^{i}=\left(\lambda^{i}, g, f\right), B^{i}=$ $\left(\lambda^{i}, a^{i}, b^{i}\right), C^{i}=\left(\lambda^{i}, g, a^{i}\right), D^{i}=\left(\lambda^{i}, f, b^{i}\right), E^{i}=\left(\lambda^{i}, g, b^{i}\right)$ and $F^{i}=\left(\lambda^{i}, f, a^{i}\right)$. Then, we have

$$
G P^{i}=A^{i} \cdot B^{i}-C^{i} \cdot D^{i}+E^{i} \cdot F^{i} .
$$

Now we consider the signs of terms appearing in $G P^{i}$. If the pivot operation $B_{i} \rightarrow B_{i+1}$ is strictly increasing, $\chi\left(A^{i}\right) \cdot \chi\left(B^{i}\right) \cdot \chi\left(C^{i}\right) \cdot \chi\left(E^{i}\right)=+1$ is satisfied. Using OM axioms, the following 12 types of signs are possible:

$$
\begin{array}{cccccc}
A^{i} \cdot B^{i}-C^{i} \cdot D^{i} & E^{i} \cdot F^{i} & \\
+ & + & + & + & + \text { type } & 1 \\
+ & + & + & + & - \text { type } & 2 \\
+ & + & - & + & - \text { type } & 3 \\
+ & - & - & - & - \text { type } & 4 \\
+ & - & - & - & + \text { type } & 5 \\
+ & - & + & - & + \text { type } & 6 \\
- & + & - & - & + \text { type } & 7 \\
- & + & - & - & - \text { type } & 8 \\
- & + & + & - & - \text { type } & 9 \\
- & - & + & + & - \text { type } & 10 \\
- & - & + & + & + \text { type } & 11 \\
- & - & - & + & + \text { type } & 12
\end{array}
$$

After normalization, type $1,4,7$ or 10 generates a biquadratic inequality $\left[E^{i}\right]\left[F^{i}\right]<\left[C^{i}\right]\left[D^{i}\right]$ and type $3,6,9$ or 12 generates a biquadratic inequality $\left[A^{i}\right]\left[B^{i}\right]<\left[C^{i}\right]\left[D^{i}\right]$. Remaining type 2, 5, 8 or 11 does not determine either of the two inequalities, but these four types cannot appear. We explain the fact by using a transition diagram in Figure 1 later.

If the pivot operation $B_{i} \rightarrow B_{i+1}$ is degenerate or horizontal, $\chi\left(A^{i}\right)$. $\chi\left(B^{i}\right)=0$ is satisfied. Using OM axioms, the following 8 types of signs are possible:

$$
\begin{array}{ccccc}
A^{i} \cdot B^{i}- & C^{i} \cdot D^{i} & +E^{i} \cdot F^{i} \\
0 & + & + & + & + \text { type } 1^{\prime} \\
0 & + & + & - & - \text { type } 2^{\prime} \\
0 & + & - & + & - \text { type } 3^{\prime} \\
0 & + & - & - & + \text { type } 4^{\prime} \\
0 & - & + & + & - \text { type } 5^{\prime} \\
0 & - & + & - & + \text { type } 6^{\prime} \\
0 & - & - & + & + \text { type } 7^{\prime} \\
0 & - & - & - & - \text { type } 8^{\prime}
\end{array}
$$


Clearly, each one of the eight types implies a biquadratic equation $\left[E^{i}\right]\left[F^{i}\right]=$ $\left[C^{i}\right]\left[D^{i}\right]$.

In both cases above, the fact $L^{i} \cup\left\{b^{i}\right\}=B_{i+1}=L^{i+1} \cup\left\{a^{i+1}\right\}$ implies the following relation:

$$
\begin{aligned}
& \chi\left(D^{i}\right) \cdot \chi\left(E^{i}\right) \cdot \chi\left(C^{i+1}\right) \cdot \chi\left(F^{i+1}\right)= \\
& \chi\left(\lambda^{i}, f, b^{i}\right) \cdot \chi\left(\lambda^{i}, g, b^{i}\right) \cdot \chi\left(\lambda^{i+1}, g, a^{i+1}\right) \cdot \chi\left(\lambda^{i+1}, f, a^{i+1}\right)=1,
\end{aligned}
$$

which restricts the types of possible successors $G P^{i+1}$ of a GrassmannPlücker relation $G P^{i}$ of certain type. The transition diagram is given in the following Figure 1. For example, if a Grassmann-Plücker polynomial $G P^{i}$ is type 3, the type of $G P^{i+1}$ is either $3,6,9,12,2^{\prime}, 3^{\prime}, 6^{\prime}, 7^{\prime}, 2,5,8$ or 11.

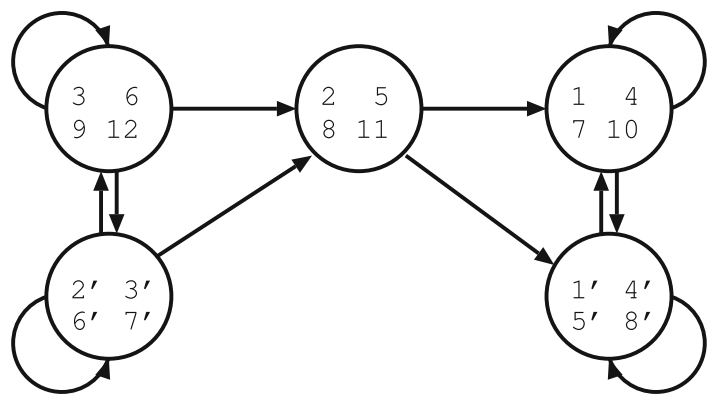

Figure 1. transition diagram among types

A Grassmann-Plücker relation of type $t$ can be succeeded by a Grassmann-Plücker relation of type $s$ if and only if there is an arrow from the circle containing $t$ to the circle containing $s$. We have $G P^{1}=G P^{k}$ and $B_{1} \rightarrow B_{2}$ is strictly increasing, that is, the type of $G P^{1}$ and $G P^{k}$ is same and either of (3). Then a sequence of transition either

- contains only two states $(1,4,7,10)$ and $\left(1^{\prime}, 4^{\prime}, 5^{\prime}, 8^{\prime}\right)$, and its initial state is $(1,4,7,10)$, or

- contains only two states $(3,6,9,12)$ and $\left(2^{\prime}, 3^{\prime}, 6^{\prime}, 7^{\prime}\right)$, and its initial state is $(3,6,9,12)$.

In both cases, the state $2,5,8$ or 11 cannot appear in the transition sequence and the resulting set of biquadratic inequalities and biquadratic equations yields a biquadratic final polynomial.

The converse of Theorem 1.4 does not hold: that is, there exist nonrealizable OMs which have a BFP but are Euclidean. For example, below the non-Pappus OM has a BFP, but is Euclidean since the rank is three. 


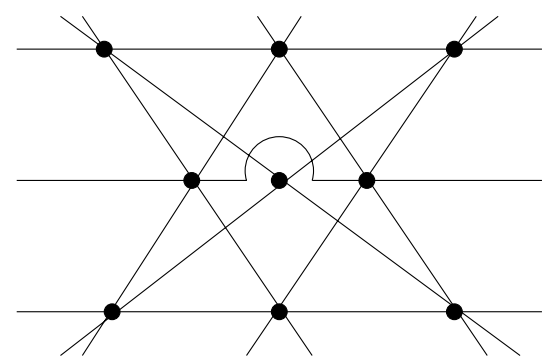

Figure 2. non-Pappus OM

\section{Historical Notes}

The main theorem, Theorem 1.4, was independently proved by the last author and by the first three authors. Although the last author's work predates the other, it was published only in the form of doctoral dissertation [5] and left unknown for many years. This led to an independent proof by the first three authors. The present paper is a natural synthesis of the two proofs.

\section{References}

[1] R. G. Bland: A combinatorial abstraction of linear programming, J. Combinatorial Theory B 23 (1977), 33-57.

[2] A. Buörner, M. Las Vergnas, B. Sturmfels, N. White and G. M. Ziegler: Oriented Matroids, Second Edition, Encyclopedia of Mathematics, Cambridge University Press (1993), 46.

[3] K. FukudA: Oriented Matroid Programming, Doctoral Thesis, University of Waterloo (1982), 223 pages.

[4] H. Nakayama, S. Moriyama, K. Fukuda and Y. Okamoto: Comparing the strength of the non-realizability certificates for oriented matroids, in: Proc. of 4 th Japanese-Hungarian Symp. on Discrete Mathematics and Its Appl., (2005), 243-249.

[5] J. Richter-Gebert: On the Realizability Problem of Combinatorial Geometries Decision Methods, Doctoral Thesis, TU-Darmstadt (1992), 144 pages.

[6] J. Richter-GeBert: Euclideaness and final polynomials in oriented matroid theory, Combinatorica 13(3) (1993), 259-268.

Komei Fukuda

Institute for Operations Research and

Institute of Theoretical Computer Science

ETH Zentrum

Zurich, CH-8092

Switzerland

fukuda@ifor.math.ethz.ch

\section{Sonoko Moriyama}

Institute for Nano Quantum

Information Electronics

University of Tokyo

7-3-1 Hongo, Bunkyo-ku

Tokyo, 113-8656

Japan

moriso@is.s.u-tokyo.ac.jp 
698 K. FUKUDA, ET AL.: EVERY NON-EUCLIDEAN ORIENTED MATROID...

\author{
Hiroki Nakayama \\ Graduate School of Information Science \\ and Technology \\ University of Tokyo \\ 7-3-1 Hongo, Bunkyo-ku \\ Tokyo, 113-8656 \\ Japan \\ nak-den@is.s.u-tokyo.ac.jp
}

\section{Jürgen Richter-Gebert}

Technische Universität München

Zentrum Mathematik (M10)

Lehrstuhl für Geometrie

und Visualisierung

Boltzmannstr. 3

Garching bei München, D-85748

Germany

richter@ma.tum.de 